axonal growth of hippocampal neurons, whereas CRMP-2$\Delta 323-381$ inhibited endogenous microtubule assembly. In addition to inducing axonal growth, CRMP-2 is also required for axonal branching.

The authors propose that CRMP-2 promotes microtubule assembly by binding and delivering tubulin heterodimers, with which it co-polymerizes, to the growing (plus) ends of microtubules. So, microtubule assembly might then affect microtubule-polymer length during axonal growth. But although CRMP-2 is a strong candidate for promoting microtubule assembly, this function alone cannot explain the role(s) of CRMP-2 in axonal differentiation and growth, as the 59-residue microtubuleassembly-promoting fragment couldn't mediate axonal growth on its own. It remains to be seen, then, what CRMP-2's other talents are.

Katrin Bussell

(2) References and links ORIGINAL RESEARCH PAPER Fukata, Y. et al. CRMP-2 binds to tubulin heterodimers to promote microtubule assembly. Nature Cell Biol. 4, 583-591 (2002)

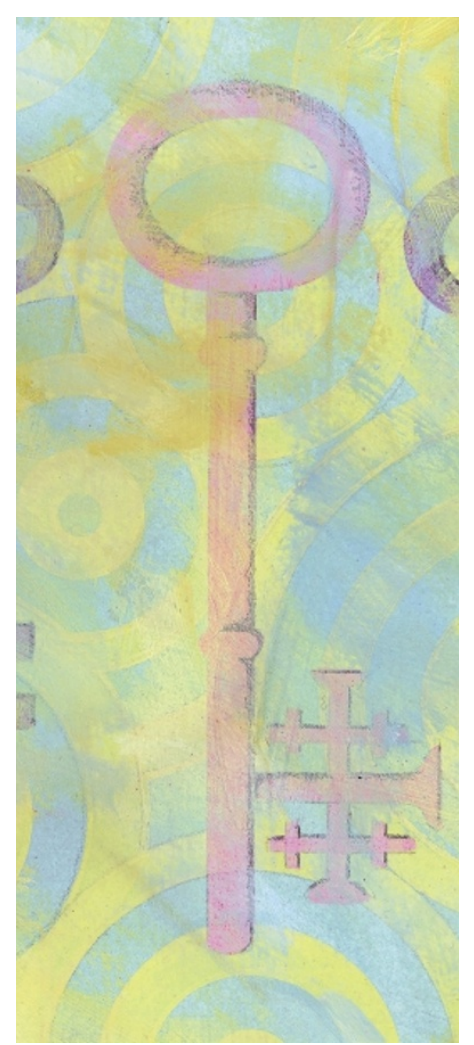

APOPTOSIS

\section{Clean debris}

Apoptotic cells are tidy - they organize their funeral while they are dying, so cellular remnants are promptly removed without any spillage of intracellular content or collateral damage. By contrast, necrotic cells become leaky, and this causes damage to neighbouring cells and inflammation. When apoptotic cells escape clearance, however, they undergo secondary necrosis. A report in Nature now describes a fail-safe mechanism in apoptotic cells that avoids inflammation after secondary necrosis, in which apoptotic cells change the structure of their chromatin, which allows it to trap a proinflammatory protein called high mobility group protein-1 (HMGB1).

HMGB1 functions as an architectural DNA-binding factor that is involved in nucleoprotein complex assembly. It also acts as a mediator of inflammation when it is secreted by activated monocytes and macrophages. Marco Bianchi and colleagues now show that these two functions are linked.

The authors first observed that HMGB1 leaks out of necrotic, but not apoptotic, cells. HMGB1 was indeed found to be tightly associated with the chromatin of apoptotic cells, even when their membrane is permeabilized artificially with detergents. As nucleosomal fragmentation is one of the hallmarks of apoptosis, the authors next tested whether DNA fragmentation is responsible for the affinity of HMGB1 for chromatin in apoptotic cells. But inhibition of DNA fragmentation did not block HMGB1 binding. Acetylation also affects chromatin structure, so they tested whether treatment with a deacetylase inhibitor before the induction of apoptosis would suppress HMGB1 binding to chromatin - and it did. This indicates that chromatin hypoacetylation occurs during apoptosis, and allows HMGB1 binding. Consistent with this histone $\mathrm{H} 4$ was found to be hypoacetylated in apoptotic cells.

What does HMGB1 binding to

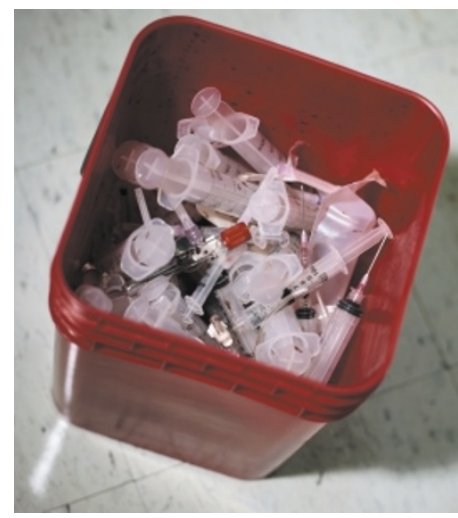

chromatin do in apoptosis? Hmgb1deficient cells are as sensitive as wildtype cells to apoptosis, which indicates that HMGB1 is not required for apoptosis. So, could HMGB1 binding to chromatin avoid its leakage and the subsequent inflammation? To test this, Bianchi and colleagues incubated bone-marrow cells with wildtype or Hmgb1-deficient dead fibroblasts. The Hmgb1-deficient necrotic cells were much less efficient than wild-type necrotic cells at eliciting the production of tumour-necrosis factor- $\alpha$ (TNF- $\alpha)$ by monocytes. Remnants from cells that had been induced to undergo secondary necrosis after prolonged exposure to an apoptotic stimulus also failed to induce any inflammation. However, remnants from fibroblasts that were treated with both TNF- $\alpha$ and a deacetylase inhibitor triggered inflammation as potently as wildtype necrotic cells.

The role of HMGB1 in inflammation after tissue necrosis was confirmed in vivo - mice that were exposed to an overdose of paracetamol undergo massive liver necrosis and subsequent inflammation. And, although anti-HMGB1 antibodies did not block liver damage, they reduced inflammation considerably. So, by changing the structure of their chromatin and tethering the proinflammatory protein HMGB1, apoptotic cells altruistically die in silence and spare their neighbours.

Valerie Ferrier, Associate Editor Nature Cell Biology

(4) References and links ORIGINAL RESEARCH PAPER Scaffidi, P.,

Misteli, T. \& Bianchi, M. E. Release of chromatin protein HMGB 1 by necrotic cells triggers inflammation. Nature 418, 191-195 (2002) WEB SITE

Marco Bianchi's laboratory: http://w3dibit.hsr.it/PhD/people/bianchi.html
IN THE NEWS

Accentuate the negative

At last! Here's a

newspaper article that understands the true plight of researchers. "The sad truth about science is that most experiments fail and the hypotheses that seduced researchers turn out not to be true or, at least, the studies provide no evidence that they are true", revealed a story in The New York Times (7 July 2002).

Bjorn Olsen at Harvard Medical School is one of many scientists who want to see these negative studies published, as they can still add to our knowledge and they would help prevent other researchers from going down the same, unsuccessful route.

Olsen is setting up an online journal, The Journal of Negative Results in Biomedicine which is expected to publish its first papers this summer. He hopes that this will overcome the difficulty of publishing studies that fail to prove hypotheses in some existing journals.

But other researchers say the attitude of journals is not the only reason for not publishing negative data. "Those [scientists] conducting the studies do not want to share them [negative data] ", Scott Kern told The New York Times.

Kern started NOGO (Negative Observations in Genetic Oncology), which focuses on negative studies of gene mutations that might predispose to cancer. This, said Kern, received great support, but few submissions. Kern suspects this is because scientists are unwilling to share this information to their peers. "They now know something they're not going to do again and their competitor does not", he said.

Simon Frantz 